\title{
Prävention - viel Gerede und wenig Taten
}

W enn man die Verlautbarungen von Gesundheitspolitikern der letzten Jahre liest, gewinnt man den Eindruck, dass Deutschland maximale Anstrengungen zur Prävention unternimmt. Dies mag für bestimmte Gebiete - insbesondere für einige Krebserkrankungen - gelten; eine Hautkrebsfrüherkennung ist aber erst seit dem Juli 2008 möglich.

Ganz besonders traurig sieht die Sache in der Allergologie aus, und wirklich problematisch wird es, wenn es um Hauterkrankungen geht. Die Fortschritte der molekularen Genetik der letzten Jahre konnten über die Ermittlung der Filaggrin-Mutationen die Natur der Barrierestörung der Epidermis bei atopischen Individuen und bei Ichthyosis vulgaris weitgehend aufklären. Daraus geht eindeutig hervor, dass einer von 400 Europäern zwei Filaggrin-Null-Mutationen aufweist und deshalb keine funktionierende epidermale Barriere aufbauen kann. Diese Patienten leiden an Ichthyosis vulgaris und haben ein hohes Ekzemrisiko. Einer von 15 Europäern weist heterozygot eine Null-Mutation auf; diese bedingt eine Neigung zur „trockenen" Haut und ein erhöhtes

„Was empirisch seit Jahrzehnten bekannt ist, dass nämlich Neurodermitiker mehr für die Pflege ihres Hautorgans tun müssen, hat eine molekulare Grundlage gewonnen."
Risiko für atopisches Ekzem. Was empirisch seit Jahrzehnten bekannt ist, dass nämlich Neurodermitiker mehr für die Pflege ihres Hautorgans tun müssen, hat damit eine molekulare Grundlage gewonnen. Der Begriff „Hautpflege“ ist dabei kontraproduktiv, da es sich in Wahrheit um die Therapie der gestörten epidermalen Barrierefunktion handelt. Wir sprechen deshalb auch lieber von „Basistherapie“ bei atopischem Ekzem.

Leider wird diese Basistherapie aber nicht mehr von den Krankenkassen getragen. Lediglich bei Kindern unter zwölf Jahren gelingt es, dies zum Teil durchzusetzen - aber auch nicht immer. Es ist von Kasse zu Kasse verschieden, welche Basistherapeutika ersetzt werden. Bei der bekannten individuellen Verträglichkeit von Emollenzien ist die Angelegenheit oft sehr schwierig. Gänzlich inakzeptabel ist die Situation für Jugendliche und Erwachsene, hier wird die Basistherapie nicht erstattet. Dabei ist dies die Grundvorausset-

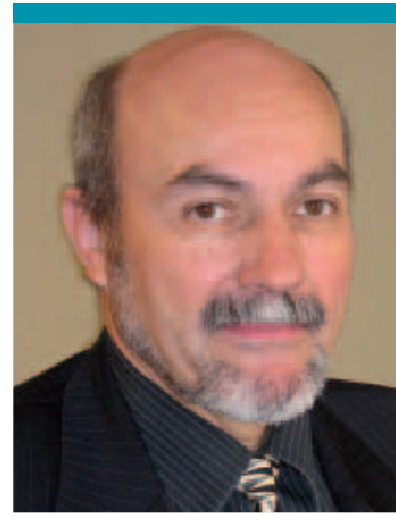

Prof. Dr. Dr. Johannes Ring, Klinik und Poliklinik für Dermatologie und Allergologie am Biederstein, Technische Universität München

zung der tertiären Prävention, nämlich der Verhinderung des nächsten Schubes.

Mit diesen Fragen wird sich auch das Programm des diesjährigen 24. Davoser Fortbildungskongresses vom 1 . bis zum 4 . Oktober befassen, dessen Abstracts Sie in diesem Heft ab Seite 455 vorab lesen können. In diesem Jahr wurde die strikte Trennung nach Symposien und Plenarvorträgen etwas aufgelockert. Der Tag wird mit den wie immer spannend ausgewählten Kasuistiken („Diaklinik“) und einem Plenum beginnen. In der zweiten Vormittagshälfte werden interaktive Seminare angeboten, in denen Experten praktisch anwendbares Wissen vermitteln. Am frühen Nachmittag wird ein zweites Plenum Fortbildung auf hohem Niveau anbieten, gefolgt wieder von praktischen Übungen und Seminaren. Ziel des Kongresses ist es, jedem Teilnehmer viele praktische Informationen zu vermitteln, die er am Montag der darauffolgenden Woche in seiner Praxis anwenden kann.

Wie in der Vergangenheit wird die $\mathrm{Ta}-$ gung auch dieses Mal Gelegenheit zur Begegnung im Sinne eines Vier-Länder-Treffens von schweizerischen, österreichischen, deutschen und Südtiroler Allergologen geben. Das Davoser Klima ist nicht nur für Patienten mit Haut- und Atemwegserkrankungen gesund, sondern auch für die Teilnehmer einer wissenschaftlichen Tagung. Wir freuen uns, Sie im Davoser Hochgebirgstal begrüßen zu dürfen!

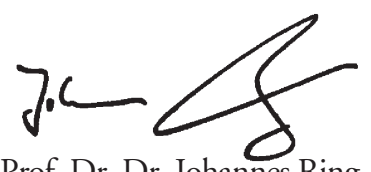

Prof. Dr. Dr. Johannes Ring 